\title{
Update: Influenza Activity — United States, October 4, 2015-February 6, 2016
}

\author{
Kate Russell, $\mathrm{MD}^{1,2}$; Lenee Blanton, $\mathrm{MPH}^{2}$; Krista Kniss, $\mathrm{MPH}^{2}$; Desiree Mustaquim, $\mathrm{MPH}^{2}$; Sophie Smith, $\mathrm{MPH}^{2}$; \\ Jessica Cohen, $\mathrm{MPH}^{2,3}$; Shikha Garg, MD²; Brendan Flannery, $\mathrm{PhD}^{2}$; Alicia M. Fry, MD²; Lisa A. Grohskopf, MD²; Joseph Bresee, MD²; \\ Teresa Wallis, $\mathrm{MS}^{2}$; Wendy Sessions, $\mathrm{MPH}^{2}$; Rebecca Garten, $\mathrm{PhD}^{2}$; Xiyan Xu, MD²; Anwar Isa Abd Elal ${ }^{2}$; Larisa Gubareva, PhD ${ }^{2}$; John $\mathrm{Barnes}^{2} \mathrm{PhD}^{2}$; \\ David E. Wentworth, $\mathrm{PhD}^{2}$; Erin Burns, $\mathrm{MA}^{2}$; Jacqueline Katz, $\mathrm{PhD}^{2}$; Daniel Jernigan, $\mathrm{MD}^{2}$; Lynnette Brammer, $\mathrm{MPH}^{2}$
}

From October through mid-December 2015, influenza activity remained low in most regions of the United States. Activity began to increase in late December 2015 and continued to increase slowly through early February 2016. Influenza A viruses have been most frequently identified, with influenza A (H3N2) viruses predominating during October until early December, and influenza A (H1N1) pdm09 viruses predominating from mid-December until early February. Most of the influenza viruses characterized during that time are antigenically similar to vaccine virus strains recommended for inclusion in the 2015-16 Northern Hemisphere vaccines. This report summarizes U.S. influenza activity* during October 4, 2015-February 6, 2016, and updates the previous summary (1).

\section{Viral Surveillance}

World Health Organization (WHO) and National Respiratory and Enteric Virus Surveillance System (NREVSS) laboratories include both public health and clinical laboratories throughout the United States and contribute to virologic surveillance for influenza. Clinical laboratories test respiratory specimens for diagnostic purposes, whereas public health laboratories primarily test specimens for surveillance purposes. Because of differences in these testing practices, virologic data for clinical and public health laboratories is being presented separately beginning with the 2015-16 influenza season.

During October 4, 2015-February 6, 2016, clinical laboratories in the United States tested 279,056 respiratory specimens for influenza viruses, of which 7,966 (2.9\%) were positive (Figure 1). During the week ending February 6 (week 5), 17,175 specimens were tested, of which 1,563 (9.1\%) were positive for influenza. Among these, 1,135 (73\%) were positive for influenza A viruses and $428(27 \%)$ were positive for influenza B viruses.

Public health laboratories tested 26,287 respiratory specimens for influenza during October 4, 2015-February 6, 2016. Of the

\footnotetext{
*The CDC influenza surveillance system collects five categories of information from nine data sources: 1) viral surveillance (U.S. World Health Organization collaborating laboratories, the National Respiratory and Enteric Virus Surveillance System, and novel influenza A virus case reporting); 2) outpatient illness surveillance (U.S. Outpatient Influenza-Like Illness Surveillance Network); 3) mortality (the National Center for Health Statistics Mortality Surveillance System, 122 Cities Mortality Reporting System, and influenza-associated pediatric mortality reports); 4) hospitalizations (FluSurv-NET, which includes the Emerging Infections Program and surveillance in three additional states); and 5) summary of the geographic spread of influenza (state and territorial epidemiologist reports).
}

3,529 specimens that were positive for influenza, 2,664 (75\%) were positive for influenza A viruses and $865(25 \%)$ were positive for influenza B viruses. Among the 2,536 (95\%) influenza A viruses subtyped, 1,698 (67\%) were influenza A (H1N1)pdm09, and $838(33 \%)$ were influenza A (H3N2) viruses. Among the influenza B viruses, 495 (57\%) had lineage determined: 372 (75\%) belonged to the B/Yamagata lineage, and 123 (25\%) belonged to the B/Victoria lineage. Since October 4, 2015, influenza-positive tests have been reported from all 50 states, the District of Columbia, and Puerto Rico, representing all U.S. Department of Health and Human Services regions. ${ }^{\dagger}$

Since October 4, age has been reported for 3,059 patients with influenza-positive tests (87\%), including 387 (13\%) children aged 0-4 years, 958 (31\%) persons aged 5-24 years, 1,294 (42\%) persons aged 25-64 years, and 420 (14\%) persons aged $\geq 65$ years. Cumulatively, influenza A (H3N2) viruses were predominant among persons aged $\geq 65$ years, whereas influenza $A(\mathrm{H} 1 \mathrm{~N} 1) \mathrm{pdm} 09$ viruses predominated among other age groups. During January 3, 2016-February 6, 2016, influenza $\mathrm{A}(\mathrm{H} 1 \mathrm{~N} 1) \mathrm{pdm} 09$ viruses have been the predominant viruses detected among all age groups. The greatest number of influenza $B$ viruses were reported in persons aged 5-24 years.

\section{Novel Influenza A Viruses}

One human infection with a novel influenza A virus was reported to CDC during the week ending January 2, 2016, (week 52) from the state of New Jersey. The patient was infected with an influenza $A(\mathrm{H} 3 \mathrm{~N} 2)$ variant ${ }^{\S}(\mathrm{H} 3 \mathrm{~N} 2 \mathrm{v})$ virus. The

\footnotetext{
$\dagger$ The 10 regions include the following jurisdictions: Region 1: Connecticut, Maine, Massachusetts, New Hampshire, Rhode Island, and Vermont; Region 2: New Jersey, New York, Puerto Rico, and the U.S. Virgin Islands; Region 3: Delaware, District of Columbia, Maryland, Pennsylvania, Virginia, and West Virginia; Region 4: Alabama, Florida, Georgia, Kentucky, Mississippi, North Carolina, South Carolina, and Tennessee; Region 5: Illinois, Indiana, Michigan, Minnesota, Ohio, and Wisconsin; Region 6: Arkansas, Louisiana, New Mexico, Oklahoma, and Texas; Region 7: Iowa, Kansas, Missouri, and Nebraska; Region 8: Colorado, Montana, North Dakota, South Dakota, Utah, and Wyoming; Region 9: Arizona, California, Hawaii, Nevada, American Samoa, Commonwealth of the Northern Mariana Islands, Federated States of Micronesia, Guam, Marshall Islands, and Republic of Palau; Region 10: Alaska, Idaho, Oregon, and Washington.

$\$$ Influenza viruses that circulate in swine are called swine influenza viruses when isolated from swine, but are called variant influenza viruses when isolated from humans. Seasonal influenza viruses that circulate worldwide in human populations have important antigenic and genetic differences from influenza viruses circulating in swine.
} 
FIGURE 1. Number* and percentage of respiratory specimens testing positive for influenza reported by clinical laboratories, by influenza virus type and surveillance week — United States, October 4, 2015-February 6, 2016

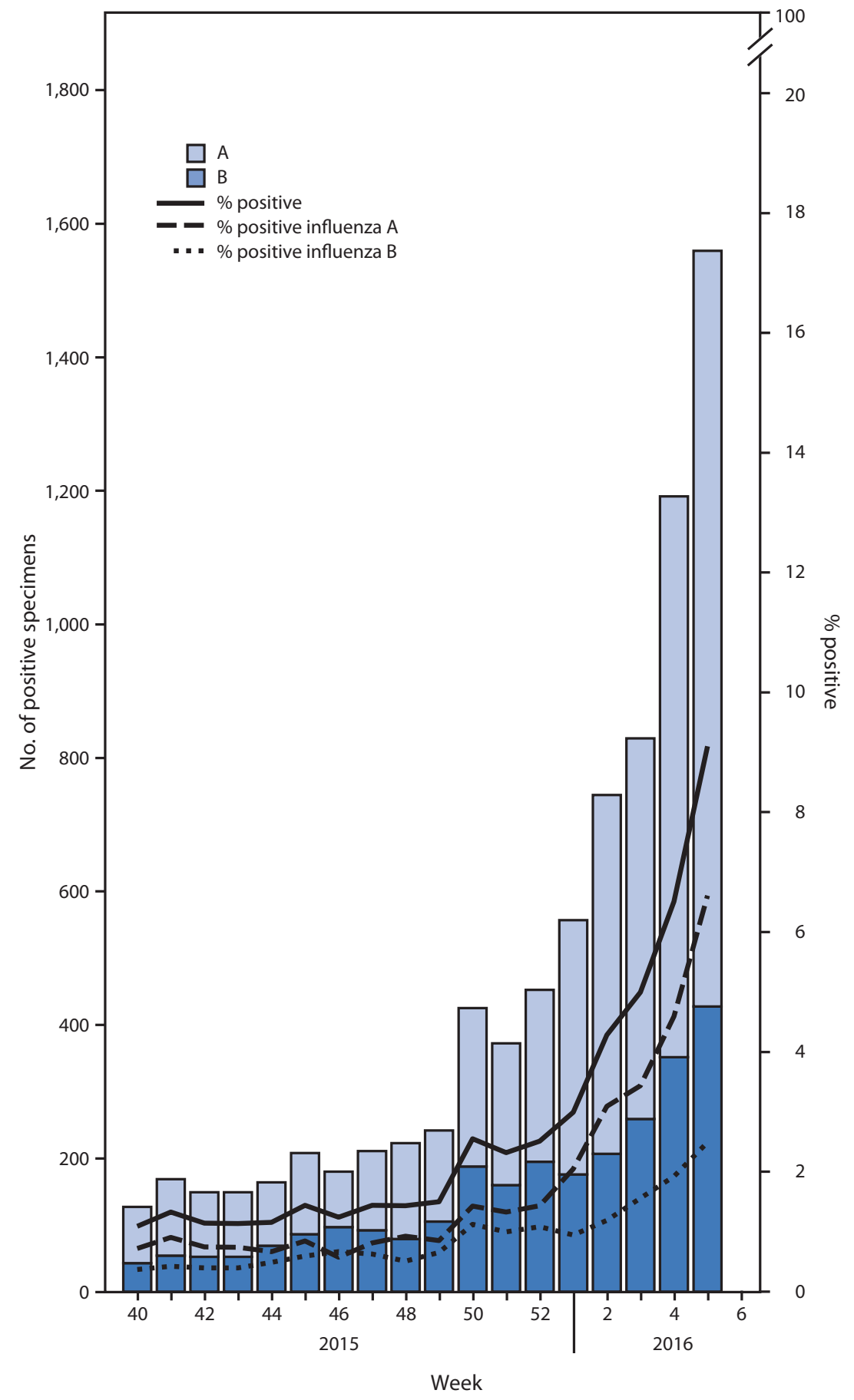

* 2,966 (2.9\%) of 279,056 tested were positive during October 4, 2015-February 6, 2016.

\section{Antigenic and Genetic Characterization of Influenza Viruses}

The 93 public health laboratories participating as WHO collaborating laboratories in the United States are requested to submit a subset of their influenza virus-positive respiratory specimens to CDC for further characterization. CDC characterizes influenza viruses through one or more laboratory tests including genome sequencing, hemagglutination inhibition (HI), or neutralization assays. These data are used to monitor circulating influenza viruses for early identification of viruses that are antigenically different from the recommended influenza vaccine reference viruses. Most viruses analyzed are propagated in mammalian cell cultures because viruses propagated in tissue culture better represent viruses in circulation, and isolation rates of human influenza viruses are higher in mammalian cell cultures than in eggs, which is the matrix used for production of the majority of influenza vaccines (2,3). In addition, viruses are more likely to undergo adaptive changes when propagated in eggs. Antigenic and genetic characterization of circulating viruses is performed using both mammalian cell- and egg-propagated reference viruses.

Data obtained from antigenic characterization continue to play an important role in the assessment of the similarity between reference viruses and circulating viruses. Although vaccine effectiveness field studies must be conducted to determine how well the vaccine is working, these laboratory data are used to evaluate whether changes in the virus that could affect vaccine effectiveness might have occurred. Beginning with the 2014-15 season, a proportion of influenza A (H3N2) viruses have not yielded sufficient hemagglutination titers for antigenic characterization by HI. For nearly all viruses characterized at CDC laboratories, next generation sequencing is performed to determine the genetic identity of circulating viruses. For the subset of viruses that do not yield sufficient hemagglutination titers, antigenic properties are inferred using results obtained from viruses within the same genetic group as those that have been characterized antigenically.

Since October 1, 2015, CDC has antigenically or genetically characterized 483 viruses from the United States 
(180 influenza A (H1N1)pdm09, 216 influenza A (H3N2), 52 influenza $\mathrm{B} /$ Yamagata lineage, and 35 influenza B/Victoria lineage). All 180 influenza A (H1N1)pdm09 viruses were antigenically characterized as A/California/7/2009-like, the influenza A (H1N1) component of 2015-16 Northern Hemisphere vaccines. Although all recent influenza $\mathrm{A}(\mathrm{H} 1 \mathrm{~N} 1) \mathrm{pdm} 09$ viruses belong to hemagglutinin (HA) genetic group $6 \mathrm{~B}$, two genetic subgroups have emerged. To date, however, both genetic subgroups remain antigenically similar to the A/California/7/2009 virus. All 216 influenza A ( $\mathrm{H} 3 \mathrm{~N} 2)$ viruses were sequenced and belonged to genetic groups for which a majority of viruses antigenically characterized were antigenically like A/Switzerland/9715293/2013, the influenza $\mathrm{A}(\mathrm{H} 3 \mathrm{~N} 2)$ reference virus representing the $\mathrm{A}(\mathrm{H} 3 \mathrm{~N} 2)$ component of the 2015-16 Northern Hemisphere vaccine. A subset of 105 influenza A (H3N2) viruses also were antigenically characterized; 98 of 105 (93\%) influenza A (H3N2) viruses were A/Switzerland/9715293/2013-like by $\mathrm{HI}$ or neutralization testing. All 52 of the $\mathrm{B} /$ Yamagata-lineage were antigenically characterized as B/Phuket/3073/2013-like, the influenza B component of the 2015-16 Northern Hemisphere trivalent and quadrivalent influenza vaccines. All 35 influenza $B$ viruses belonging to the $\mathrm{B} /$ Victoria-lineage were antigenically characterized as $\mathrm{B} /$ Brisbane/60/2008-like, an influenza B component of the 2015-16 Northern Hemisphere quadrivalent influenza vaccines.

\section{Antiviral Resistance of Influenza Viruses}

Since October 4, 2015, a total of 699 influenza viruses (301 influenza A (H1N1)pdm09 viruses, 246 influenza A (H3N2) viruses, and 152 influenza $B$ viruses) have been examined for antiviral resistance by the WHO Collaborating Center for Surveillance, Epidemiology, and Control of Influenza at CDC. All 152 influenza B viruses and 246 influenza A (H3N2) viruses tested were sensitive to oseltamivir and peramivir. Among 301 influenza A (H1N1)pdm09 viruses tested for resistance, two $(0.7 \%)$ were found to be resistant to both oseltamivir and peramivir. All 301 influenza A (H1N1)pdm09 viruses tested were sensitive to zanamivir. High levels of resistance to the adamantanes (amantadine and rimantadine) persist among influenza $\mathrm{A}$ (H1N1)pdm09 and (H3N2) viruses. Adamantane drugs are not recommended for use against influenza at this time.

\section{Outpatient IIIness Surveillance}

Since October 4, 2015, the weekly percentage of outpatient visits for influenza-like illness (ILI) ${ }^{* *}$ reported by approximately

\footnotetext{
"A virus is considered "reference virus-like" if its hemagglutination inhibition (HI) or neutralization focus reduction (FRA) titer is within 4-fold of the homologous $\mathrm{HI} / \mathrm{FRA}$ titer of the reference strain. A virus is considered as low to the reference virus if there is $\geq 8$-fold or greater reduction in the HI or FRA titer when compared with the homologous HI or FRA titer of the reference strain.

** Defined as a fever $\geq 100^{\circ} \mathrm{F}\left(\geq 37.8^{\circ} \mathrm{C}\right)$, oral or equivalent, and cough and/or sore throat, without a known cause other than influenza.
}

2,000 U.S. Outpatient ILI Surveillance Network (ILINet) providers in 50 states, New York City, Chicago, the U.S. Virgin Islands, Puerto Rico, and the District of Columbia that constitute ILINet has ranged from $1.3 \%-2.5 \%$. The percentage exceeded the national baseline ${ }^{\dagger \dagger}$ of $2.1 \%$ for 2 consecutive weeks, from the week ending December 26, 2015-January 2, 2016 (weeks 51 and 52) (Figure 2). The increase in percentage of patient visits for ILI during those 2 weeks might be influenced in part by a reduction in routine health care visits during the winter holiday season, as has occurred during previous influenza seasons. The percentage was at or above the national baseline for 4 consecutive weeks, from the week ending January 16, 2016-February 6, 2016 (weeks 2-5). During the 1997-1998 through 2014-15 influenza seasons, excluding the 2009 pandemic, peak weekly percentages of outpatient visits for ILI ranged from $2.4 \%-7.7 \%$ and remained above baseline levels for an average of 13 weeks (range $=1-19$ weeks). For the week ending February 6, 2016 (week 5), the percentage of outpatient visits for ILI was $2.4 \%$, and seven U.S. Department of Health and Human Services regions (1, 2, 3, 4, 6, 8, and 10) reported ILI activity at or above region-specific baseline levels.

Data collected in ILINet are used to produce a measure of ILI activity $\$ \mathbb{S}$ by jurisdiction. During the week ending February 6 , 2016 (week 5), Puerto Rico and one state (Arizona) experienced high ILI activity. Two states (Arkansas and Connecticut) experienced moderate ILI activity. New York City and eight states (Florida, Illinois, Massachusetts, New Mexico, Oklahoma, Oregon, Texas, and Utah) experienced low ILI activity. Minimal ILI activity was experienced in 38 states (Alabama, Alaska, California, Delaware, Georgia, Hawaii, Idaho, Indiana, Iowa, Kansas, Kentucky, Louisiana, Maine, Maryland, Michigan, Minnesota, Mississippi, Missouri, Montana, Nebraska, Nevada, New Hampshire, New Jersey, New York, North Carolina, North Dakota, Ohio, Pennsylvania, Rhode Island, South Carolina, South Dakota, Tennessee, Vermont, Virginia, Washington, West Virginia, Wisconsin, and Wyoming). The District of Columbia and one state (Colorado) had insufficient data to report.

\footnotetext{
$\dagger \dagger$ The national and regional baselines are the mean percentage of visits for ILI during noninfluenza weeks for the previous three seasons plus two standard deviations. Noninfluenza weeks are defined as periods of $\geq 2$ consecutive weeks in which each week accounted for $<2 \%$ of the season's total number of specimens that tested positive for influenza. National and regional percentages of patient visits for ILI are weighted on the basis of state population. Use of the national baseline for regional data is not appropriate.

$\$ \mathbb{S}$ Activity levels are based on the percentage of outpatient visits in a jurisdiction attributed to ILI and are compared with the average percentage of ILI visits that occur during weeks with little or no influenza virus circulation. Activity levels range from minimal, corresponding to ILI activity from outpatient clinics at or below the average, to high, corresponding to ILI activity from outpatient clinics much higher than the average. Because the clinical definition of ILI is nonspecific, not all ILI is caused by influenza; however, when combined with laboratory data, the information on ILI activity provides a clearer picture of influenza activity in the United States.
} 
FIGURE 2. Percentage of visits for influenza-like illness (ILI)* reported to the CDC, by surveillance week - Outpatient Influenza-Like IIIness Surveillance Network, United States, 2015-16 influenza season and selected previous influenza seasons

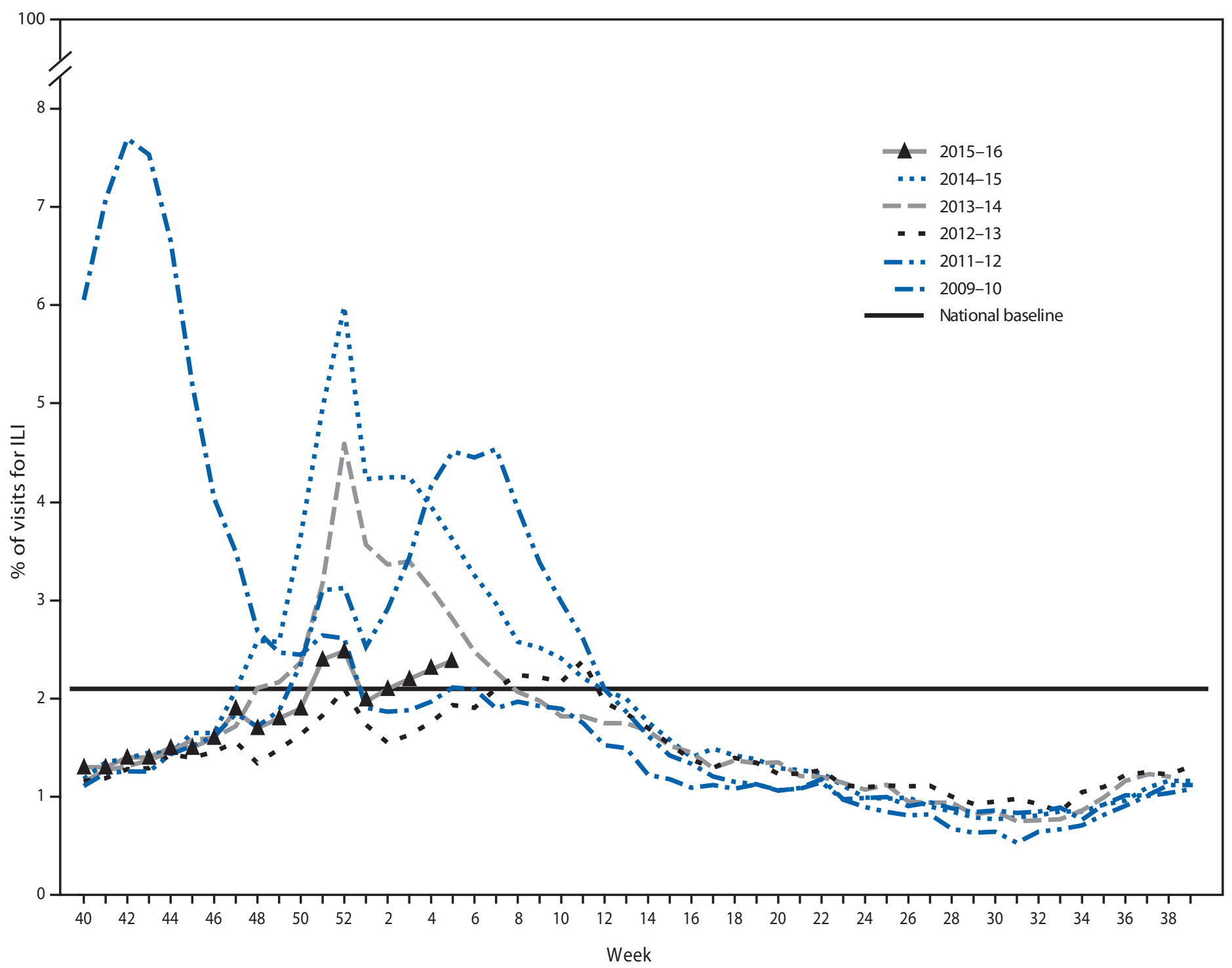

* Defined as fever $\left(\geq 100^{\circ} \mathrm{F}\left[\geq 37.8^{\circ} \mathrm{C}\right]\right)$, oral or equivalent, and cough and/or sore throat, without a known cause other than influenza.

\section{Geographic Spread of Influenza}

For the week ending February 6 (week 5), Puerto Rico and seven states (Arizona, California, Connecticut, Iowa, Kentucky, Massachusetts, and New York) reported widespread activity. 99

\footnotetext{
99 Levels of activity are 1) no activity; 2) sporadic: isolated laboratory-confirmed influenza cases or a laboratory-confirmed outbreak in one institution, with no increase in activity; 3) local: increased ILI, or two or more institutional outbreaks (ILI or laboratory-confirmed influenza) in one region of the state, with recent laboratory evidence of influenza in that region; virus activity no greater than sporadic in other regions; 4) regional: increased ILI activity or institutional outbreaks (ILI or laboratory-confirmed influenza) in more than two, but less than half of the regions in the state with recent laboratory evidence of influenza in those regions; and 5) widespread: increased ILI activity or institutional outbreaks (ILI or laboratory-confirmed influenza) in at least half the regions in the state, with recent laboratory evidence of influenza in the state.
}

Guam and 17 states (Florida, Indiana, Maine, Maryland, Michigan, Minnesota, Nevada, New Hampshire, New Jersey, New Mexico, North Dakota, Pennsylvania, Rhode Island, Texas, Utah, Vermont, and Washington) reported regional activity. Sixteen states (Alabama, Arkansas, Colorado, Idaho, Illinois, Kansas, Montana, North Carolina, Ohio, Oklahoma, Oregon, South Carolina, Tennessee, Virginia, Wisconsin, and Wyoming) reported local activity and the District of Columbia and nine states (Alaska, Delaware, Georgia, Hawaii, Louisiana, Missouri, Nebraska, South Dakota, and West Virginia) reported sporadic activity. No activity was reported in one state (Mississippi) and the U.S. Virgin Islands did not report. During the previous five influenza seasons, the peak number of jurisdictions reporting 
widespread activity during each season has ranged from 20 in the 2011-12 season to 49 in the 2010-11 season.

\section{Influenza-Associated Hospitalizations}

CDC monitors hospitalizations associated with laboratoryconfirmed influenza infection in adults and children through the Influenza Hospitalization Surveillance Network (FluSurvNET), ${ }^{* * *}$ which covers approximately 27 million persons, 9\% of the U.S. population. During October 4, 2015-February 6, 2016, a total of 896 laboratory-confirmed influenza-associated hospitalizations were reported, with a cumulative incidence for all age groups of 3.2 per 100,000 . Persons aged $\geq 65$ years had the highest rate of influenza-associated hospitalization and accounted for approximately $40 \%$ of reported influenza-associated hospitalizations. The cumulative hospitalizations rate (per 100,000 population) during October 4, 2015-February 6, 2016, was 4.5 among children aged $<5$ years, 1.1 among children and adolescents aged 5-17 years, 1.5 among adults aged 18-49 years, 4.1 among adults aged 50-64 years and 10.2 among adults aged $\geq 65$ years. During the past three influenza seasons (2012-13 through 2014-15), end-of-season age-specific cumulative hospitalization rates have ranged from 47.3-67.0 per 100,000 population for persons aged $0-4$ years, $9.4-16.6$ for persons aged 5-17 years, $16.1-21.4$ for persons aged $18-49$ years, 40.9-53.7 for persons aged 50-64 years, and 84.7-308.5 for persons aged $\geq 65$ years. Among all hospitalizations reported during October 4 , 2015-February 6, 2016, a total of $624(70 \%)$ were associated with influenza A, 242 (27\%) with influenza B, 20 (2.2\%) with influenza $A$ and $B$ co-infection, and 10 (1.1\%) had no virus type information. Among 189 patients with influenza A subtype information, $160(85 \%)$ were $\mathrm{A}(\mathrm{H} 1 \mathrm{~N} 1) \mathrm{pdm} 09$ virus and 29 $(15 \%)$ were $\mathrm{A}(\mathrm{H} 3 \mathrm{~N} 2)$ virus.

\footnotetext{
*** FluSurv-NET conducts population-based surveillance for laboratoryconfirmed influenza-associated hospitalizations in children and adolescents aged $<18$ years (since the $2003-04$ influenza season) and adults aged $\geq 18$ years (since the 2005-06 influenza season). The FluSurv-NET covers approximately 70 counties in the 10 Emerging Infections Program states (California, Colorado, Connecticut, Georgia, Maryland, Minnesota, New Mexico, New York, Oregon, and Tennessee) and additional Influenza Hospitalization Surveillance Project (IHSP) states. IHSP began during the 2009-10 season to enhance surveillance during the $2009 \mathrm{H} 1 \mathrm{~N} 1$ pandemic. IHSP sites included Iowa, Idaho, Michigan, Oklahoma, and South Dakota during the 2009-10 season; Idaho, Michigan, Ohio, Oklahoma, Rhode Island, and Utah during the 2010-11 season; Michigan, Ohio, Rhode Island, and Utah during the 2011-12 season; and Iowa, Michigan, Ohio, Rhode Island, and Utah during the 2012-13 season; and Michigan, Ohio, and Utah during the 2013-14, 2014-15, and 2015-16 seasons. Cumulative unadjusted incidence rates are calculated using CDC's National Center for Health Statistics population estimates for the counties included in the surveillance catchment area. Laboratory confirmation is dependent on clinician-ordered influenza testing, and testing for influenza often is underutilized because of the poor reliability of rapid test results and greater reliance on clinical diagnosis for influenza. As a consequence, cases identified as part of influenza hospitalization surveillance likely are an underestimation of the actual number of persons hospitalized with influenza.
}

Complete medical chart abstraction data were available for 349 (39\%) hospitalized patients with laboratory-confirmed influenza as of February 6, 2016. Among these, 91\% of hospitalized adults had at least one underlying medical condition that placed them at high risk for influenza-associated complications. ${ }^{\dagger \dagger}$ The most commonly reported medical conditions were cardiovascular disease (39\%), metabolic disorders (38\%), and obesity (36\%). Forty seven percent of hospitalized children had at least one underlying medical condition, the most commonly reported being asthma (19\%) and neurologic disorders (17\%). Among 29 hospitalized women of childbearing age (15-44 years), 7 (24\%) were pregnant.

\section{Pneumonia and Influenza-Associated Mortality}

Pneumonia and influenza (P\&I)-associated deaths are tracked through two systems, the National Center for Health Statistics (NCHS) Mortality Surveillance System, which reports the week the death occurred, and the 122 Cities Mortality Reporting System, which reports the week that the death certificate was registered. Because of these differences in reporting, the two data sources produce different percentages. Beginning with the 2015-16 influenza season, the NCHS Mortality Surveillance System has been the principal component of U.S. Mortality Surveillance System.

For the week ending January 23, 2016 (week 3), 6.9\% $(1,861$ of 27,158$)$ of all U.S. deaths were classified as resulting from P\&I as reported by NCHS (Figure 3). This percentage is below the epidemic threshold of $7.6 \%$ for week $3 . \$ \$ \$$ Since October 4, 2015 the percentage of deaths attributable to P\&I ranged from $6.2 \%$ to $7.2 \%$ and has not exceeded the epidemic

\footnotetext{
it† Persons at higher risk include children aged $<5$ years (especially those aged $<2$ years); adults aged $\geq 65$ years; persons with chronic pulmonary (including asthma), cardiovascular (except hypertension alone), renal, hepatic, hematologic (including sickle cell disease), metabolic disorders (including diabetes mellitus), or neurologic and neurodevelopment conditions (including disorders of the brain, spinal cord, peripheral nerve, and muscle, such as cerebral palsy, epilepsy [seizure disorders], stroke, intellectual disability [mental retardation], moderate to severe developmental delay, muscular dystrophy, or spinal cord injury); persons with immunosuppression, including that caused by medications or by human immunodeficiency virus infection; women who are pregnant or postpartum (within 2 weeks after delivery); persons aged $\leq 18$ years who are receiving long-term aspirin therapy; American Indians/Alaska Natives; persons who are morbidly obese (i.e., body mass index $\geq 40$ ); and residents of nursing homes and other chronic care facilities.

$\$ \$ \$$ The seasonal baseline proportion of P\&I deaths is projected using a robust regression procedure, in which a periodic regression model is applied to the observed percentage of deaths from P\&I that were reported by the National Center for Health Statistics Mortality Surveillance System and the 122 Cities Mortality Reporting System during the preceding 5 years. The epidemic threshold is set at 1.645 standard deviations above the seasonal baseline. Users of the data should not expect the NCHS mortality surveillance data and the 122 Cities Mortality Reporting System to produce the same percentages, and the percent P\&I deaths from each system should be compared with the corresponding system specific baselines and thresholds.
} 
FIGURE 3. Percentage of all deaths attributable to pneumonia and influenza (P\&I), by surveillance week and year* — National Center for Health Statistics Mortality Surveillance System, United States, 2012-2016

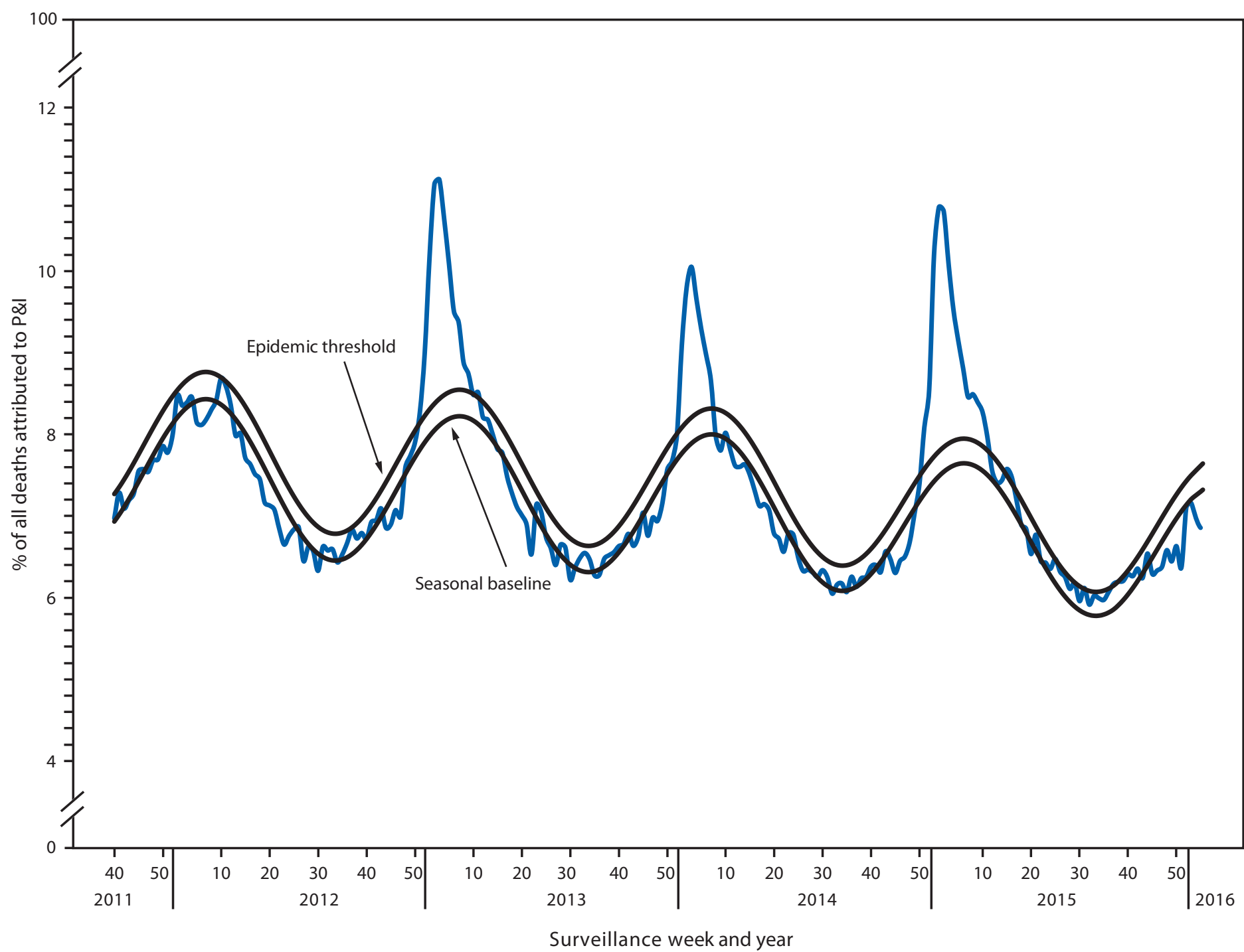

* Data as of February 6, 2016.

threshold this season. During the past five influenza seasons, peak weekly percentages of deaths attributable to P\&I have ranged from $8.7 \%$ during the $2011-12$ season to $11.1 \%$ during the 2012-13 season.

Since October 4, 2015, the weekly percentage of deaths attributed to P\&I as reported in the 122 Cities Mortality Reporting System has not exceeded the epidemic threshold for $\geq 2$ weeks, ranging from $5.2 \%-7.7 \%$. For the week ending February 6, 2016 (week 5), the weekly percentage of deaths attributable to P\&I was $6.2 \%$, below the epidemic threshold of $6.9 \%$ for week 5 . During the past five influenza seasons, peak weekly percentages of deaths attributable to P\&I have ranged from $7.8 \%$ during the $2011-12$ season to $9.9 \%$ during the $2012-13$ season.

\section{Influenza-Associated Pediatric Mortality}

As of February 6, 2016 (week 5), 11 influenza-associated pediatric deaths that occurred during the 2015-16 season have been reported to CDC. Of these, one death was associated with an influenza $\mathrm{A}(\mathrm{H} 3 \mathrm{~N} 2)$ virus, three were associated with an influenza $\mathrm{A}(\mathrm{H} 1 \mathrm{~N} 1) \mathrm{pdm} 09$ virus, three were associated with an influenza $\mathrm{A}$ virus for which no subtyping was performed, and four were associated with an influenza B virus. Since influenza-associated pediatric mortality became nationally notifiable in 2004 , the total number of influenza-associated pediatric deaths has ranged from $37-171$ per season, excluding the 2009 pandemic, during which 358 pediatric deaths were reported to CDC during April 15, 2009-October 2, 2010. 


\section{Discussion}

Timing of influenza activity in the United States can be variable but most often peaks during January-March (4). During the three most recent influenza seasons, 2012-13, 2013-14, and 2014-15, activity began relatively early, and peaked in late December and early January. The current season activity began to increase in mid-December, a more typical influenza activity pattern. Activity has continued to increase through February 6, 2016. It is not possible to predict when influenza activity will peak but influenza activity will likely continue to increase and remain elevated for several weeks. Influenza A (H3N2), influenza $A(\mathrm{H} 1 \mathrm{~N} 1) \mathrm{pdm} 09$, and influenza $\mathrm{B}$ viruses have cocirculated this season. During the weeks ending October 10, 2015-December 5, 2015 (weeks 40 through 48), influenza A (H3N2) was the most common virus identified. However, beginning with week 49, influenza A (H1N1)pdm09 has been the most common. CDC has received reports of severe respiratory illness among young- to middle-aged adults with influenza $\mathrm{A}$ (H1N1)pdm09 (5). This has also been observed during previous seasons when influenza A (H1N1)pdm09 predominated (G).

Although vaccine effectiveness estimates are not yet available for the 2015-16 Northern Hemisphere vaccine, laboratory data to date have indicated similarity between circulating viruses and recommended vaccine components. Vaccination remains the best way to prevent influenza infection and associated complications (4). Health care providers should continue to offer and encourage vaccination for unvaccinated persons aged $\geq 6$ months throughout the influenza season.

Although influenza vaccination is the best way to prevent influenza, antiviral medications are an important adjunct for reducing the health impact of influenza. Treatment with influenza antiviral medications as early as possible is recommended for patients with confirmed or suspected influenza who have severe, complicated, or progressive illness; who require hospitalization; or who are at high risk for influenzarelated complications $(7,8)$. Antiviral treatment should not be withheld from high-risk or severely ill patients with suspected influenza infection pending confirmatory influenza text results or based on illness onset ( 7 ). Treatment is most effective when given early in the illness; providers should not delay treatment while waiting for test results and should not rely on insensitive assays such as rapid antigen detection influenza diagnostic tests to determine treatment $(7,8)$.

Influenza surveillance reports for the United States are posted online weekly (http://www.cdc.gov/flu/weekly). Additional information regarding influenza viruses, influenza surveillance, influenza vaccine, influenza antiviral medications, and novel influenza A infections in humans is online (http://www.cdc.gov/flu).

\section{Summary}

What is already known about this topic?

CDC collects, compiles, and analyzes data on influenza activity year-round in the United States. Timing of influenza activity and predominant circulating influenza viruses vary by season.

What is added by this report?

Influenza activity remained low in the United States through early December and began to increase slowly in mid-December. Influenza A and $B$ viruses have been reported. Influenza A (H3N2) viruses predominated from October to mid-December, and influenza A (H1N1)pdm09 viruses have predominated from mid-December to February. To date, the majority of influenza viruses that have been antigenically or genetically characterized are similar to components of the 2015-16 Northern Hemisphere vaccine.

What are the implications for public health practice?

Vaccination is the primary method to prevent influenza illness and its complications. Health care providers should continue to recommend influenza vaccination to all unvaccinated persons aged $\geq 6$ months now and throughout the influenza season. As an adjunct to vaccine, treatment with influenza antiviral medications is recommended for patients with confirmed or suspected influenza who have severe, complicated, or progressive illness; who require hospitalization; or who are at high risk for influenza-related complications. Antivirals can lessen severity and duration of illness and can reduce severe outcomes of influenza. Antiviral medications work best when administered early in the course of influenza-like illness.

\section{Acknowledgments}

State, county, city, and territorial health departments and public health laboratories; U.S. World Health Organization collaborating laboratories; National Respiratory and Enteric Virus Surveillance System laboratories; U.S. Outpatient Influenza-Like Illness Surveillance Network sites; FluSurv-NET; National Center for Health Statistics, CDC; 122 Cities Mortality Reporting System; World Health Organization, FluNet; Angie Foust, Elisabeth Blanchard, Priya Budhathoki, Thomas Rowe, Lizheng Guo, Ewelina Lyszkowicz, Shoshona Le, Malania Wilson, Juliana DaSilva, Alma Trujillo, Michael Hillman, Thomas Stark, Samuel Shepard, Sujatha Seenu, Ha Nguyen, Vasiliy Mishin, Margaret Okomo-Adhiambo, Michelle Adamczyk, Juan De la Cruz, Influenza Division, National Center for Immunization and Respiratory Diseases, CDC.

\footnotetext{
${ }^{1}$ Epidemic Intelligence Service, CDC; ${ }^{2}$ Influenza Division, National Center for Immunization and Respiratory Diseases, CDC; ${ }^{3}$ Atlanta Research and Education Foundation, Georgia.

Corresponding author: Kate Russell, KERussell@cdc.gov, 404-639-3747.
} 


\section{References}

1. Smith S, Blanton L, Kniss K, et al. Update: influenza activity-United States. MMWR Morb Mortal Wkly Rep 2015;64:1342-8. http://dx.doi. org/10.15585/mmwr.mm6448a4.

2. Schild GC, Oxford JS, de Jong JC, Webster RG. Evidence for host-cell selection of influenza virus antigenic variants. Nature 1983;303:706-9. http://dx.doi.org/10.1038/303706a0.

3. Katz JM, Wang M, Webster RG. Direct sequencing of the HA gene of influenza $(\mathrm{H} 3 \mathrm{~N} 2)$ virus in original clinical samples reveals sequence identity with mammalian cell-grown virus. J Virol 1990;64:1808-11.

4. Fiore AE, Uyeki TM, Broder K, et al. Prevention and control of influenza with vaccines: recommendations of the Advisory Committee on Immunization Practices (ACIP), 2010. MMWR Recomm Rep 2010;59(No. RR-8).
5. CDC. Health Alert Network No. 387-Flu season begins: severe influenza illness reported. Atlanta, GA: US Department of Health and Human Services, CDC; 2016. http://emergency.cdc.gov/han/han00387.asp.

6. Epperson S, Blanton L, Kniss K, et al. Influenza activity-United States, 2013-14 season and composition of the 2014-15 influenza vaccines. MMWR Morb Mortal Wkly Rep 2014;63:483-90.

7. Fiore AE, Fry A, Shay D, Gubareva L, Bresee JS, Uyeki TM. Antiviral agents for the treatment and chemoprophylaxis of influenzarecommendations of the Advisory Committee on Immunization Practices (ACIP). MMWR Recomm Rep 2011;60(No. RR-1).

8. Dobson J, Whitley RJ, Pocock S, Monto AS. Oseltamivir treatment for influenza in adults: a meta-analysis of randomised controlled trials. Lancet 2015;385:1729-37. http://dx.doi.org/10.1016/S0140-6736(14)62449-1. 